\title{
Basic fibroblast growth factor increases IFT88 expression in chondrocytes
}

\author{
DAOLU ZHAN ${ }^{1,2}$, WEI XIANG ${ }^{3}$, FENGJING GUO $^{3 *}$ and YUANZHENG MA ${ }^{1,2^{*}}$ \\ ${ }^{1}$ Graduate School, Southern Medical University, Guangzhou, Guangdong 510515; ${ }^{2}$ Department of Orthopedics, \\ The 309th Hospital of Chinese PLA, Beijing 100091; ${ }^{3}$ Department of Orthopedics, Tongji Hospital, \\ Tongji Medical College, Huazhong University of Science and Technology, Wuhan, Hubei 430030, P.R. China
}

Received November 21, 2016; Accepted July 11, 2017

DOI: $10.3892 / \mathrm{mmr} .2017 .7449$

\begin{abstract}
Intraflagellar transport protein 88 (IFT88) is protein crucial for the assembly and maintenance of primary cilia in chondrocytes. Primary cilia regulate mechanical and chemical signals in chondrocytes; however, the effects of cytokines on IFT88 expression and cilia formation and maintenance remain to be elucidated. Therefore, the role of basic fibroblast growth factor (bFGF) on IFT88 expression were examined in theATDC5 murine chondrocytic line, in order to investigate the signaling pathways involved in this process. bFGF treatment upregulated IFT88 expression in a dose- and time-dependent manner in ATDC5 cells. The effects of bFGF on IFT88 protein expression were suppressed in the presence of the extracellular signal-regulated protein kinase (ERK) inhibitor PD0325901 and the FGF receptor inhibitor BGJ398. In addition, treatment with IFT88-trageting small interfering (si)RNA downregulated the protein expression of IFT88 and ERK, thus suggesting that the ERK signaling pathway may be involved in the regulation of IFT88 expression in ATDC5 cells. bFGF treatment increased the number of ciliated ATDC5 cells and primary cultured chondrocytes. Downregulation of IFT88 expression by PD0325901, BGJ398, or IFT88-targeting siRNA was revealed to reduce the number of ciliated cells. bFGF also upregulated the mRNA and protein expression of IFT88 in
\end{abstract}

Correspondence to: Professor Yuanzheng Ma, Department of Orthopedics, The 309th Hospital of Chinese PLA, 17 Heishanhu Road, Haidian, Beijing 100091, P.R. China

E-mail:myzdr@sina.com

Professor Fengjing Guo, Department of Orthopedics, Tongji Hospital, Tongji Medical College, Huazhong University of Science and Technology, 1095 Jiefang Avenue, Wuhan, Hubei 430030, P.R. China

E-mail: fjguo@tjh.tjmu.edu.cn

${ }^{*}$ Contributed equally

Key words: chondrocyte, basic fibroblast growth factor, intraflagellar transport protein 88 , ERK signaling pathway, primary cilia primary cultured chondrocytes. In conclusion, the findings of the present study suggested that bFGF may enhance the expression of IFT88, and promote primary cilia development, through the mitogen-activated protein kinase/ERK-mediated pathway in chondrocytes.

\section{Introduction}

Primary cilia are microtubule-based organelles that protrude from most eukaryotic cells (1). Primary cilia consist of nine pairs of peripheral microtubules organized in a ring without a central microtubule. The outer peripheral microtubule structure is collectively termed the $9+0$ axoneme $(2,3)$. With the exception of cells undergoing division, most eukaryotic cells have a single primary cilium (4,5). Primary cilia were believed to have no important functions in normal cells; however, previous studies have indicated that they can act as sensory receptors for mechanical and chemical stimuli, and they may transmit these signals intracellularly (6-8).

The intraflagellar transport (IFT) complex consists of $>20$ different IFT proteins that assemble into macromolecular complexes, which can be transported through the action of kinesin or dynein motors along the flagellar axoneme $(9,10)$. IFTs are responsible for the assembly and maintenance of cilia and flagella in eukaryotic cells $(9,11)$. Intraflagellar transport protein 88 (IFT88) is a central component of the IFT-B complex, and is required for ciliogenesis in vertebrates and for flagellar assembly in Chlamydomonas $(12,13)$. IFT88 has a critical role in cilia formation; however, the regulation of IFT88 expression in chondrocytes remains to be fully elucidated.

Basic fibroblast growth factor (bFGF) is a member of the FGF family with mitogenic properties, which may exert numerous functions and a wide range of effects in cells. bFGF may bind heparin and heparin sulfate (14) and regulate the migration, differentiation, proliferation and survival of various types of cells (15-17). bFGF is also involved in the regulation of articular cartilage homeostasis; however, the exact mechanical and biochemical processes involved in cartilage degeneration and the function of bFGF in these processes remain to be fully elucidated. In addition, the effects of bFGF on IFT88 expression and the formation and maintenance of cilia in chondrocytes remain unclear. Therefore, the present 
study aimed to identify the effects of bFGF on IFT88 expression and primary cilia formation in chondrocytes in vitro.

\section{Materials and methods}

Cells and reagents. Murine ATDC5 chondrogenic cells were purchased from the Type Culture Collection of the Chinese Academy of Sciences (Shanghai, China). The cells were plated at a density of $1 \times 10^{4}$ cells $/ \mathrm{cm}^{2}$ and cultured in Dulbecco's modified Eagle's medium/nutrient mixture F12 (DMEM/F12; Hyclone; GE Healthcare Life Sciences, Logan, UT, USA) supplemented with $5 \%$ fetal bovine serum (FBS), penicillin (100 U/ml) and streptomycin $(100 \mathrm{U} / \mathrm{ml})$. Cells were maintained at $37^{\circ} \mathrm{C}$ in a $5 \% \mathrm{CO}_{2}$ atmosphere. Media were replaced every other day.

Primary cultured chondrocytes were prepared from the femoral cartilage of 6 male C57BL mice (age, 2 weeks; weight, $8.85 \pm 1.07 \mathrm{~g}$ ). Mice were maintained in an environment with a $12 \mathrm{~h} \mathrm{light/dark} \mathrm{cycle} \mathrm{and} \mathrm{at} 23 \pm 1^{\circ} \mathrm{C}$ and with free access to food and water. All mice were supplied by the Experimental Animal Center of Tongji Hospital (Wuhan, China) and the procedures were approved by the Ethics Committee on Animal Experimentation of Tongji Medical College, Huazhong University of Science and Technology (Wuhan, China). The cartilage was dissected, enzymatically digested with $0.25 \%$ trypsin solution (Sigma-Aldrich; Merck KGaA, Darmstadt, Germany) at $37^{\circ} \mathrm{C}$ for $30 \mathrm{~min}$ and $0.1 \%$ type I collagenase solution (Invitrogen; Thermo Fisher Scientific, Inc., Waltham, MA, USA) at $37^{\circ} \mathrm{C}$ for $6 \mathrm{~h}$. The single cell suspensions that were obtained were resuspended in DMEM/F12 supplemented with $10 \%$ FBS following washing in phosphate-buffered saline (PBS).

bFGF was purchased from Cyagen Biosciences Inc. (Santa Clara, CA, USA), PD0325901 and BGJ398 were purchased from Selleck Chemicals (Houston, TX, USA). ATDC5 cells were treated at $37^{\circ} \mathrm{C}$ with different concentrations of bFGF $(0,2.5,5,10,20$ or $50 \mathrm{ng} / \mathrm{ml}$ for $24 \mathrm{~h})$ and for different durations ( $5 \mathrm{ng} / \mathrm{ml}$ for $0,12,24,36,48$ or $72 \mathrm{~h}$ ). The control group received a DMSO vehicle treatment. The ERK and FGFR inhibitors PD0325901 (11 nM) and BGJ398 (1 nM), respectively, were used to treated cells at $37^{\circ} \mathrm{C}$ for $24 \mathrm{~h}$.

Western blot analysis. ATDC5 cells were lysed in radioimmunoprecipitation assay lysis buffer (Wuhan Boster Biological Technology, Ltd., Wuhan, China) containing a protease inhibitor cocktail. Extracted protein concentrations were measured using a bicinchoninic acid assay and equal quantities $(20 \mu \mathrm{g})$ were separated by $10 \%$ SDS-PAGE and transferred onto polyvinylidene difluoride membranes. The proteins were then blocked with 5\% BSA (Biosharp, Hefei, China) at $37^{\circ} \mathrm{C}$ for $1 \mathrm{~h}$. Blots were incubated at $4^{\circ} \mathrm{C}$ overnight with the following primary antibodies: IFT88 (AP11138b, 1:1,000 dilution; Abgent, Inc., San Diego, CA, USA), $\beta$-actin (BM0627, 1:400 dilution; Wuhan Boster Biological Technology, Ltd.), extracellular signal-regulated protein kinase (ERK, \#4695; 1:1,000 dilution), and phosphorylated (p)-ERK (\#4370, 1:1,000 dilution) (both from Cell Signaling Technology, Inc., Danvers, MA, USA), followed by incubation with horseradish peroxidase-conjugated goat anti-rabbit or goat anti-mouse immunoglobulin (Ig)G (BA1054 and BA1050,
1:5,000 dilution; Wuhan Boster Biological Technology, Ltd.) secondary antibodies at $37^{\circ} \mathrm{C}$ for $1 \mathrm{~h}$. An enhanced chemiluminescence (ECL) western blot detection kit (Thermo Fisher Scientific, Inc.) was used to visualize the protein bands on an ECL system (Bio-Rad Laboratories, Inc., Hercules, CA, USA). Blots were semi-quantified by densitometric analysis using Image-Lab software version 4.0.1 (Bio-Rad Laboratories, Inc.).

Reverse transcription-quantitative polymerase chain reaction $(R T-q P C R)$. Total RNA was extracted from cells following incubation using the RNeasy Mini kit (Qiagen GmbH, Hilden, Germany), according to the manufacturer's protocol. cDNA was synthesized from total RNA using the SuperScript First-Strand Synthesis system for RT-PCR (Invitrogen; Thermo Fisher Scientific, Inc.), according to the manufacturer's protocol. qPCR was performed on cDNA using a SYBR Green Real-Time PCR Master Mix kit (Toyobo Life Science, Osaka, Japan). The thermocycling conditions were as follows: Pre-denaturation at $95^{\circ} \mathrm{C}$ for $1 \mathrm{~min}$, followed by 40 cycles of denaturation at $95^{\circ} \mathrm{C}$ for $15 \mathrm{sec}$, annealing at $60^{\circ} \mathrm{C}$ for $15 \mathrm{sec}$ and extension at $72^{\circ} \mathrm{C}$ for $45 \mathrm{sec}$. Gene expression levels were normalized to those of $\beta$-actin and the method of quantification was $2^{-\Delta \Delta \mathrm{Cq}}(18)$. The primer sequences used in the present study were as follows: IFT88 forward, (F) 5'-TGGCCAACG ACCTGGAGATTAACA-3' and reverse, (R) 5'-ATAGCTGCT GGCTTGGGCAAATTC-3'; and $\beta$-actin F, 5'-CTTCTTGGG TATGGAATCCTGTGG-3' and R, 5'-TGTGTTGGCATA GAGGTCTTTACG-3'.

Small interfering (si)RNA transfection. ATDC5 cells $\left(1 \times 10^{6}\right)$ were transfected with $50 \mathrm{nM}$ siRNA targeting IFT88 or with a scrambled sequence (negative control siRNA) for $72 \mathrm{~h}$ using Lipofectamine 2000 (Invitrogen; Thermo Fisher Scientific, Inc.) as the transfection reagent. The knockdown efficiency of IFT88 siRNA was assessed using RT-qPCR and western blot analysis, as aforementioned. IFT88 siRNA and negative control siRNA (siG151225113820 and siN05815122147) were synthesized by Guangzhou RiboBio Co., Ltd. (Guangzhou, China).

Immunofluorescence. ATDC5 cells were seeded on coverslips at a density of $5 \times 10^{3}$ cells $/ \mathrm{cm}^{2}$ and stimulated with the various reagents. The cells were fixed in $4 \%$ paraformaldehyde for 15 min following $24 \mathrm{~h}$ of treatment, blocked with $0.5 \%$ bovine serum albumin (Biosharp) at room temperature for $1 \mathrm{~h}$, and then incubated with a primary acetylated $\alpha$-tubulin (acTub) antibody (ab24610, 1:500 dilution; Abcam, Cambridge, UK) overnight at $4^{\circ} \mathrm{C}$. The cells were then incubated with cyanine 3-conjugated goat anti-mouse IgG secondary antibody (BA1031, 1:200 dilution; Wuhan Boster Biological Technology, Ltd.) at room temperature for $1 \mathrm{~h}$, and the nuclei were stained with $1 \mu \mathrm{g} / \mu \mathrm{l}$ DAPI at $37^{\circ} \mathrm{C}$ for $5 \mathrm{~min}$. The cells were washed with PBS 3 times for 10 min after each step. Stained cells were visualized under a fluorescence microscope and photomicrographs were captured using an EVOS FL Auto Cell Imaging System (Thermo Fisher Scientific, Inc.).

Statistical analysis. Each experiment was performed at least 3 times. Data are presented as the mean \pm standard deviation. 
A

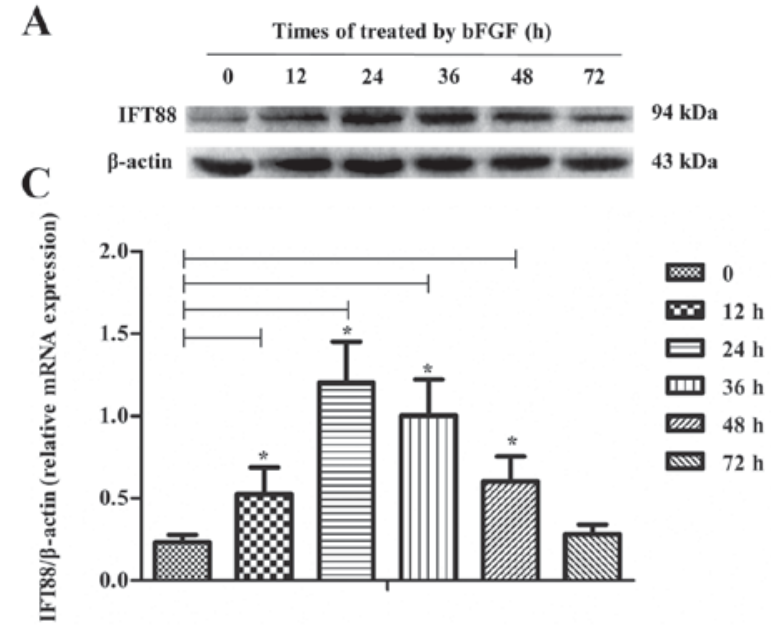

$\mathbf{E}$

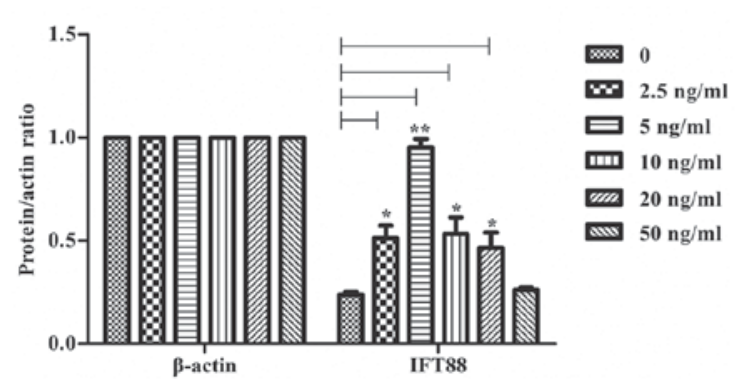

B

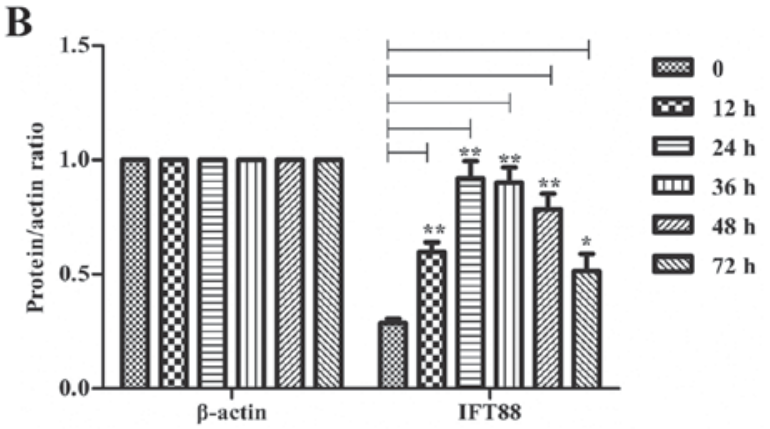

D

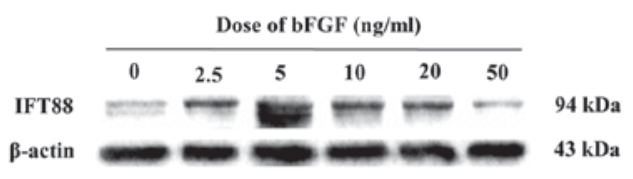

F

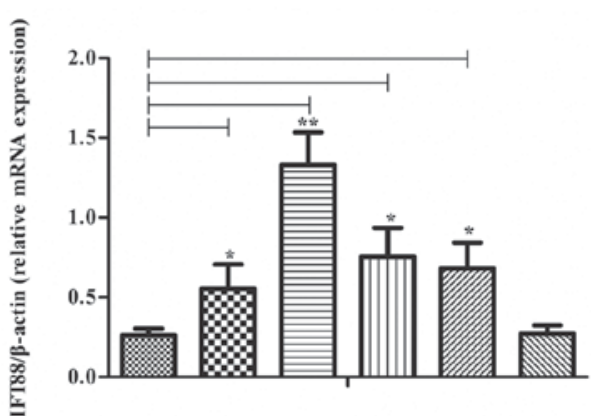

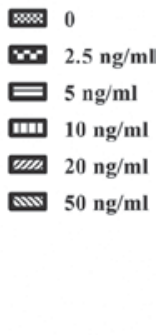

Figure 1. bFGF treatment enhanced the mRNA and protein expression of IFT88 in a time- and dose-dependent manner in murine ATDC5 chondrocytes in vitro. (A-C) ATDC5 cells were cultured for the indicated times $(0,12,24,36,48$ and $72 \mathrm{~h}$ ) in the presence of bFGF (5 ng/ml) or (D-F) with the indicated concentrations of bFGF $(0,2.5,5,10,20$ and $50 \mathrm{ng} / \mathrm{ml})$ for $24 \mathrm{~h}$. (A, B, D, E) IFT88 protein expression levels were assessed using western blot analysis with $\beta$-actin as a loading control. (C and F) IFT88 mRNA expression was detected using reverse transcription-quantitative polymerase chain reaction and normalized to $\beta$-actin mRNA levels. Data are expressed as the mean \pm standard deviation of 3 independent experiments. ${ }^{*} \mathrm{P}<0.05$, ${ }^{* *} \mathrm{P}<0.01$, as indicated. bFGF, basic fibroblast growth factor; IFT88, intraflagellar transport protein 88
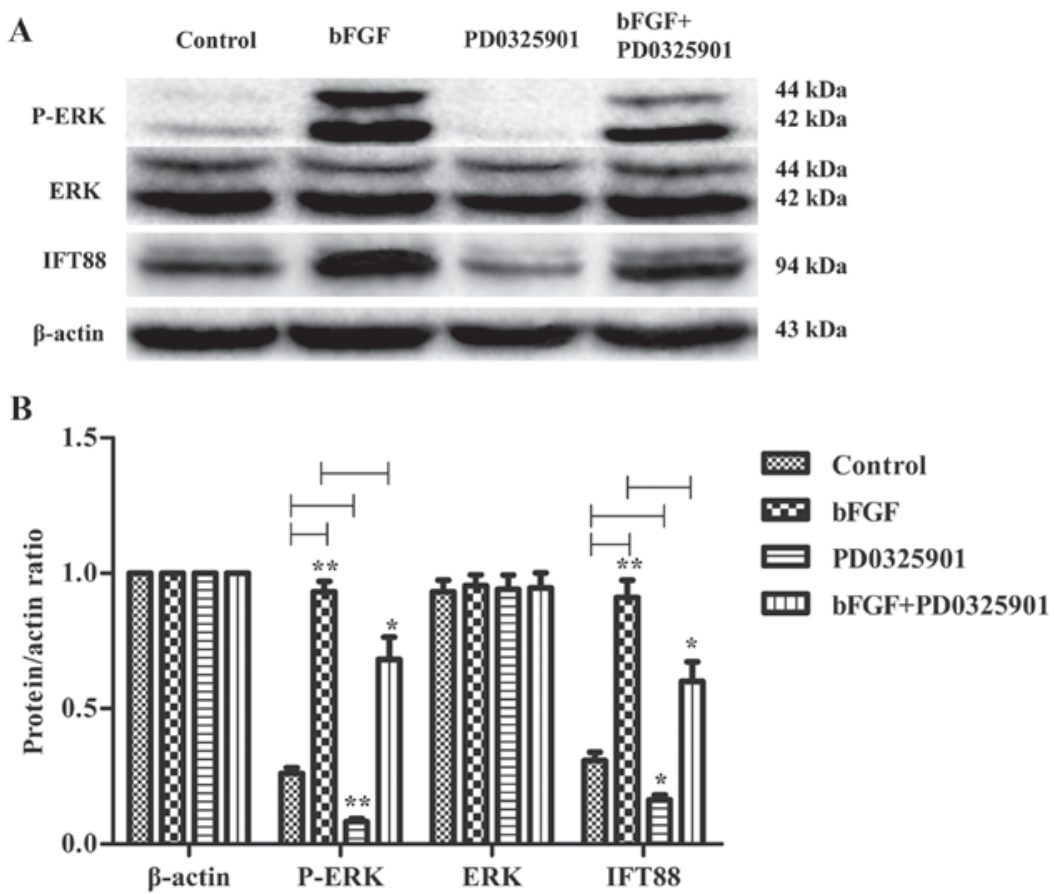

Figure 2. Treatment with the ERK inhibitor PD0325901 attenuated the effects of bFGF on the protein expression of IFT88 in murine ATDC5 chondrocytes. ATDC5 cells were treated with bFGF in the presence or absence of $11.0 \mathrm{nM}$ PD0325901. (A) Protein expression of ERK, p-ERK and IFT88 was detected using western blot analysis. $\beta$-actin was used as the loading control. (B) Blots were semi-quantified by densitometry. Data are expressed as the mean \pm standard deviation. ${ }^{*} \mathrm{P}<0.05,{ }^{* *} \mathrm{P}<0.01$, as indicated. ERK, extracellular signal-regulated protein kinase; p-ERK, phosphorylated-ERK; bFGF, basic fibroblast growth factor; IFT88, intraflagellar transport protein 88 . 
A

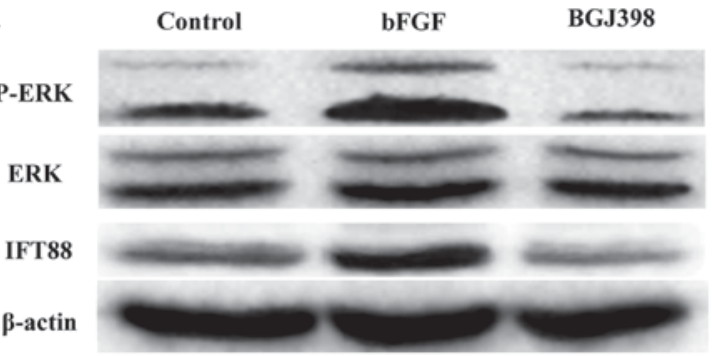

44 kDa

$42 \mathrm{kD}$

$44 \mathrm{kDa}$

$42 \mathrm{kDa}$

$94 \mathrm{kDa}$

$43 \mathrm{kDa}$

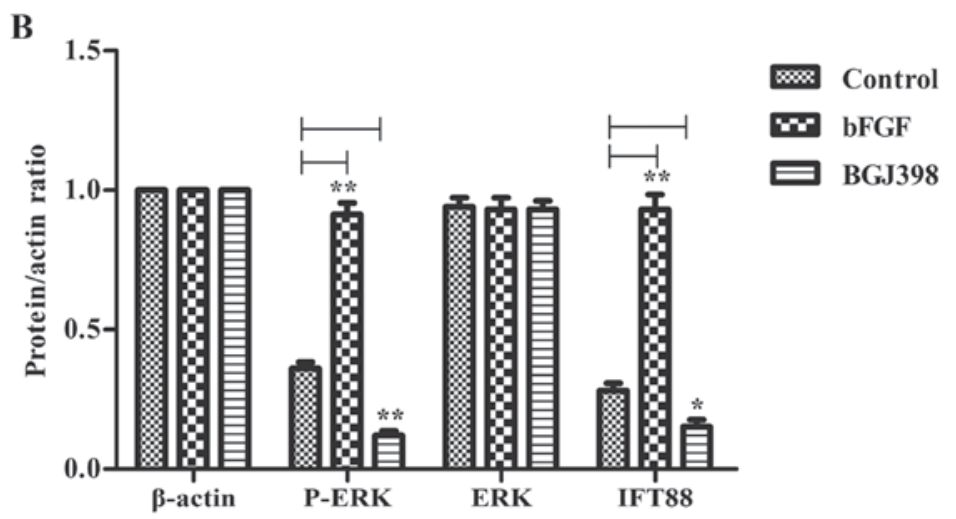

Figure 3. Treatment with the FGFR inhibitor BGJ398 downregulated IFT88 and p-ERK protein expression in murine ATDC5 chondrocytes in vitro. ATDC5 cells were cultured in the presence of $5 \mathrm{ng} / \mathrm{ml}$ bFGF or $1 \mathrm{nM} \mathrm{BGJ398.} \mathrm{(A)} \mathrm{The} \mathrm{protein} \mathrm{expression} \mathrm{levels} \mathrm{of} \mathrm{IFT88,} \mathrm{ERK} \mathrm{and} \mathrm{p-ERK} \mathrm{were} \mathrm{detected} \mathrm{using} \mathrm{western}$ blot analysis with $\beta$-actin as a loading control. (B) Blots were semi-quantified by densitometry. Data are expressed as the mean \pm standard deviation. ${ }^{*} \mathrm{P}<0.05$, ${ }^{* *} \mathrm{P}<0.01$, as indicated. FGF, fibroblast growth factor; FGFR, FGF receptor; IFT88, intraflagellar transport protein 88; ERK, extracellular signal-regulated protein kinase; p-ERK, phosphorylated-ERK; bFGF, basic FGF.

A

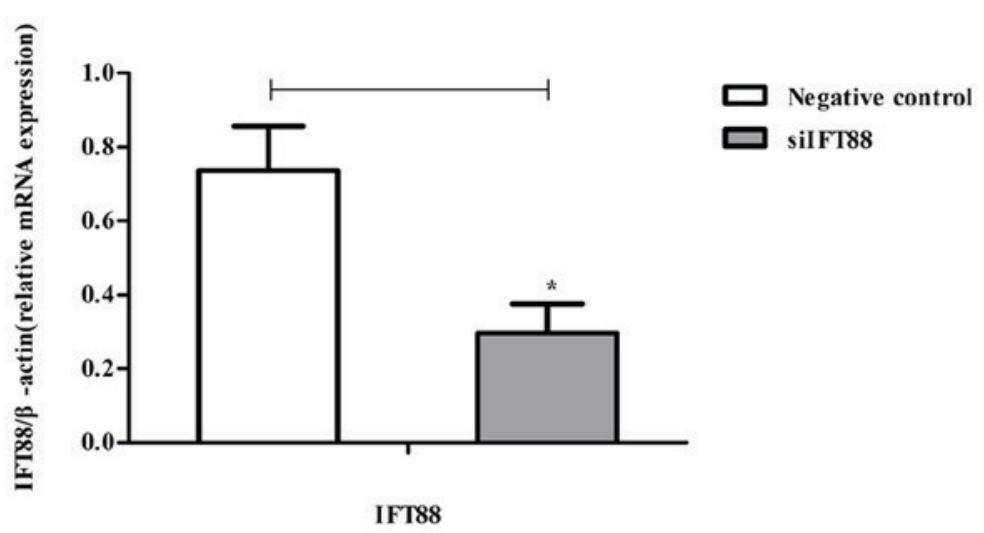

B

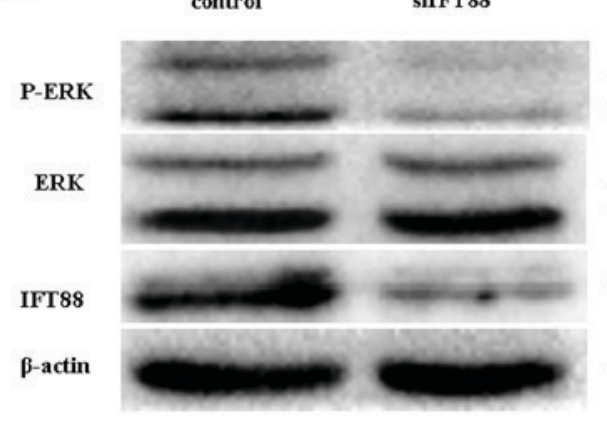

C

$44 \mathrm{kDa}$
$42 \mathrm{kDa}$
$44 \mathrm{kDa}$
$42 \mathrm{kDa}$
$94 \mathrm{kDa}$
$43 \mathrm{kDa}$

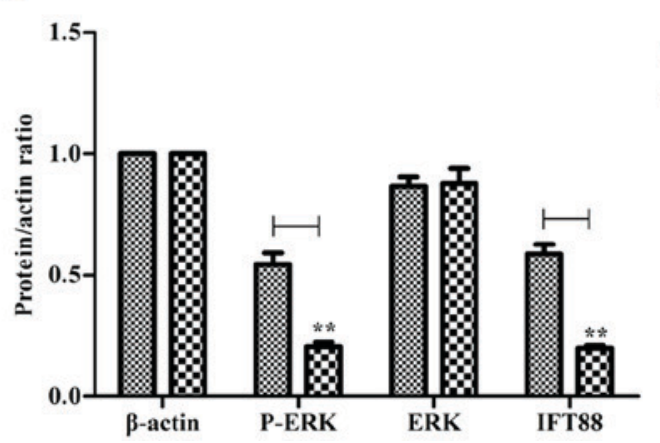

Figure 4. IFT88 knockdown suppresses the mRNA and protein expression of IFT88 in ATDC5 cells. ATDC5 cells were transfected with control siRNA or IFT88-targeting siRNA and cultured for $72 \mathrm{~h}$. (A) The efficacy of transfection was confirmed using reverse transcription-quantitative polymerase chain reaction analysis of IFT88 mRNA expression. (B and C) The protein expression levels of IFT88, p-ERK and ERK were detected using western blot analysis with $\beta$-actin as a loading control. Data are expressed as the mean \pm standard deviation of 3 independent experiments. "P<0.05, ${ }^{* *} \mathrm{P}<0.01$, as indicated. IFT88, intraflagellar transport protein 88; si, small interfering; ERK, extracellular signal-regulated protein kinase; p-ERK, phosphorylated-ERK. 
A
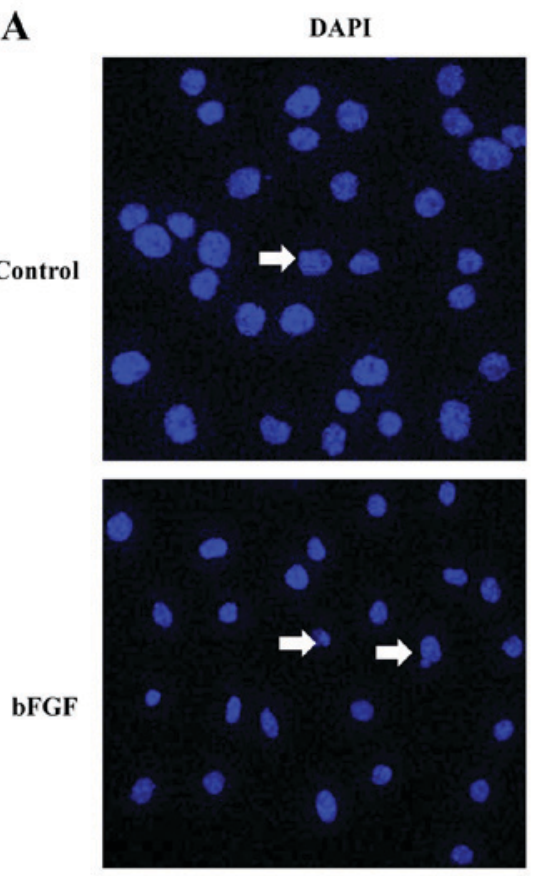

Acetylated- $\alpha$-tubulin
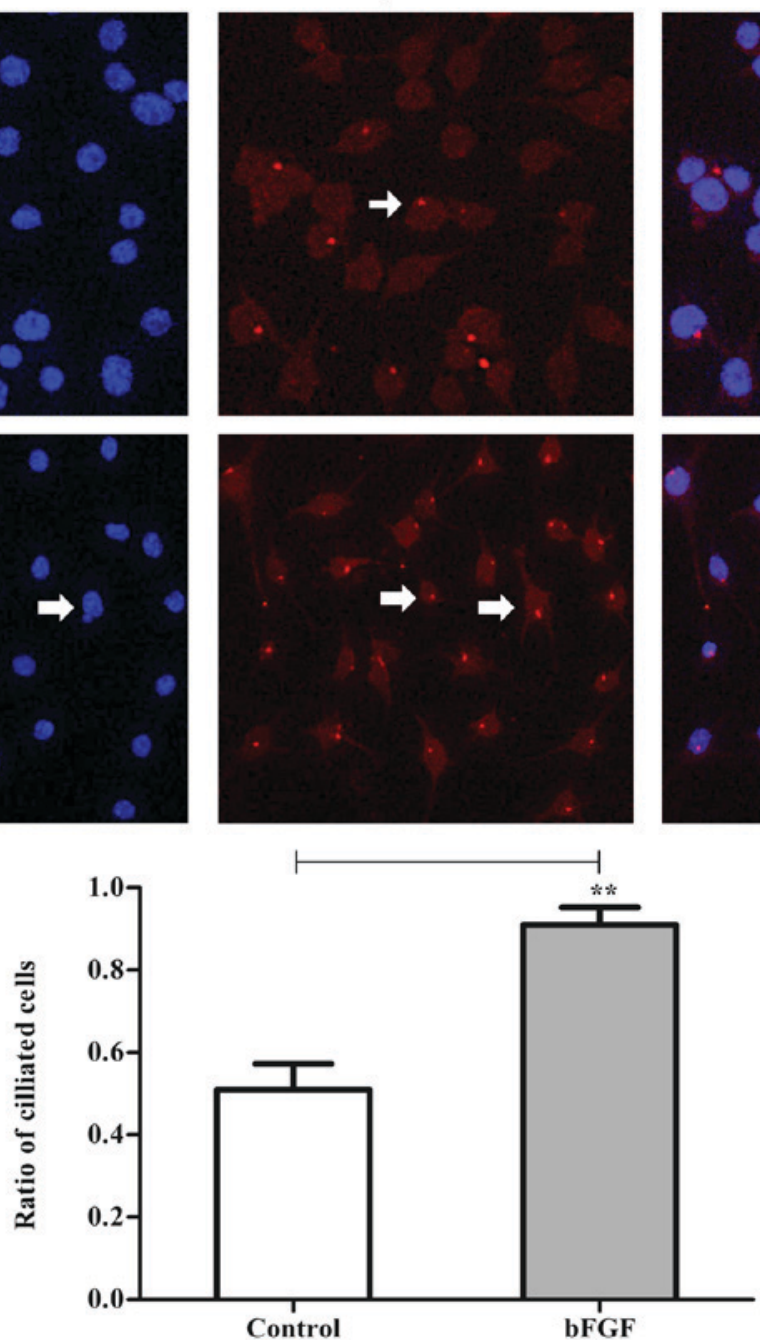

Merged
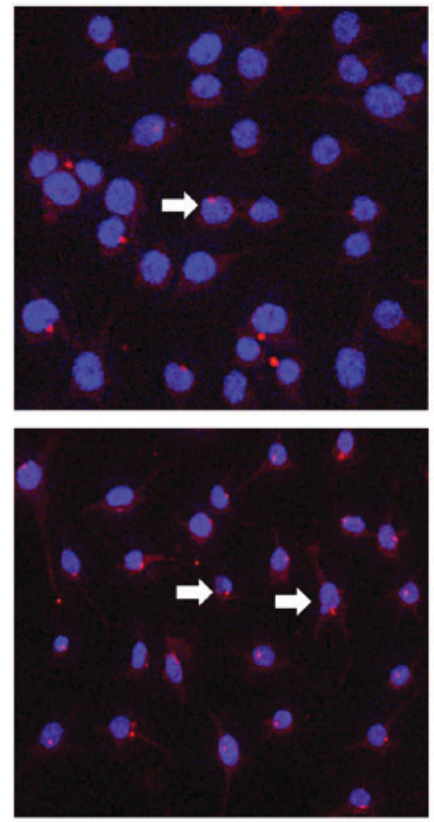

B

Figure 5. bFGF treatment increases the number of ciliated murine ATDC5 chondrocytes in vitro. ATDC5 cells were cultured in the presence or absence of $5 \mathrm{ng} / \mathrm{ml} \mathrm{bFGF}$ for $24 \mathrm{~h}$ prior to staining with an anti-acTub (red) antibody. DAPI was used to visualize the nuclei (blue). (A) bFGF treatment led to increased primary cilium (white arrows) development compared with the control group. Magnification, x200. (B) The percentage of ciliated cells was determined in 10 randomly selected fields of view, and $>100$ cells/well were counted. Data are expressed as the mean \pm standard deviation. ${ }^{* * *} \mathrm{P}<0.01$, as indicated. bFGF, basic fibroblast growth factor; acTub, acetylated $\alpha$-tubulin.

The statistical significance of the differences between groups was assessed using Student's t-test and two-way analysis of variance followed by a Bonferroni post-hoc test were used. $\mathrm{P}<0.05$ was considered to indicate a statistically significant difference. All statistical analyses were performed using SPSS version 20.0 (IBM Corporation, Armonk, NY, USA).

\section{Results}

bFGF treatment increasesIFT88 expression in a time- and dose-dependent manner in ATDC5 cells. To examine the effects of bFGF on IFT88 mRNA and protein expression levels, ATDC 5 cells were treated with $5 \mathrm{ng} / \mathrm{ml} \mathrm{bFGF}$ for up to $72 \mathrm{~h}$. bFGF treatment was demonstrated to upregulate the mRNA and protein expression of IFT 88 within $12 \mathrm{~h}$, a maximal effect was evident following $24 \mathrm{~h}$ of treatment; the effects of bFGF continued for $72 \mathrm{~h}$ (Fig. 1A-C). To investigate whether the effects of bFGF on IFT88 expression were dose-dependent, RT-qPCR and western blot analysis were performed using ATDC5 cells treated with various doses of bFGF. ATDC5 cells were treated with bFGF at concentrations of $0,2.5,5,10,20$, and $50 \mathrm{ng} / \mathrm{ml}$ for $24 \mathrm{~h}$. bFGF treatment was revealed to potentiate the protein expression of IFT88 in a dose-dependent manner; this effect was evident at $2.5 \mathrm{ng} / \mathrm{ml}$ and peaked at $5 \mathrm{ng} / \mathrm{ml}$ (Fig. 1D and E). The effect of bFGF on IFT88 protein expression gradually decreased at concentrations $>5 \mathrm{ng} / \mathrm{ml}$, but expression remained above the IFT 88 levels in control cells, with the exception of cells treated with $50 \mathrm{ng} / \mathrm{ml} \mathrm{bFGF} \mathrm{(Fig.} \mathrm{1D} \mathrm{and} \mathrm{E).} \mathrm{In} \mathrm{addition,} \mathrm{bFGF} \mathrm{treatment}$ increased the mRNA expression of IFT88 in a dose-dependent manner, and the results were similar to those regarding protein expression (Fig. 1F).

ERK inhibition counteracts the effect of bFGF on IFT88 protein expression. To investigate the putative signaling pathways implicated in the regulatory effects of bFGF on IFT88 expression, ATDC5 cells were treated with bFGF in the presence or absence of the ERK inhibitor PD0325901. The 
A
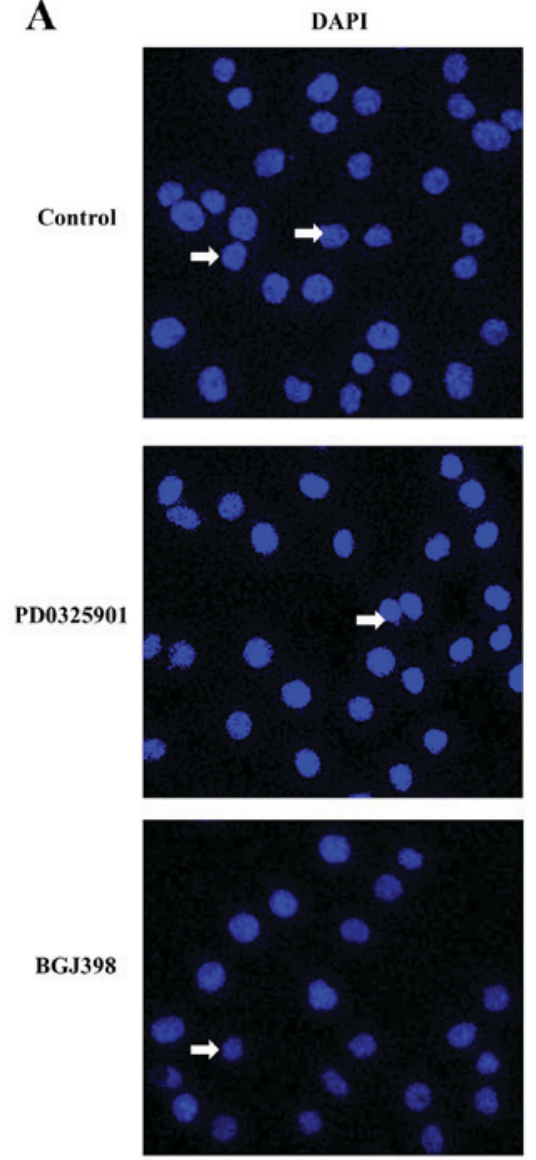

B
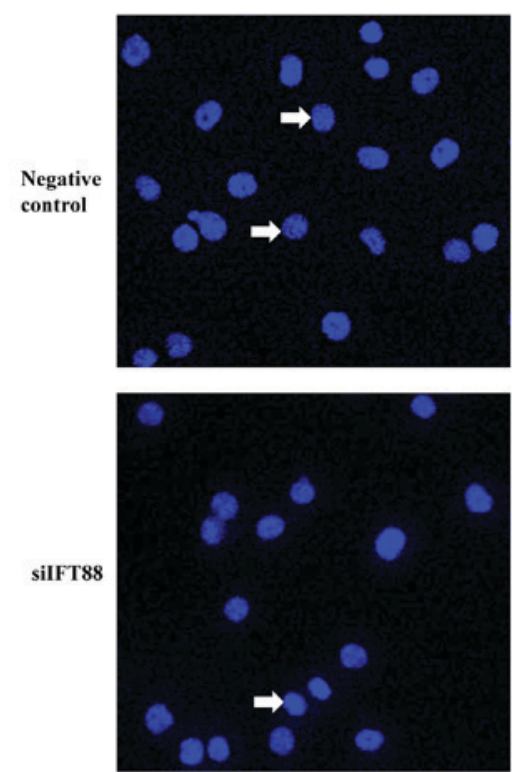

Acetylated- $\alpha$-tubulin
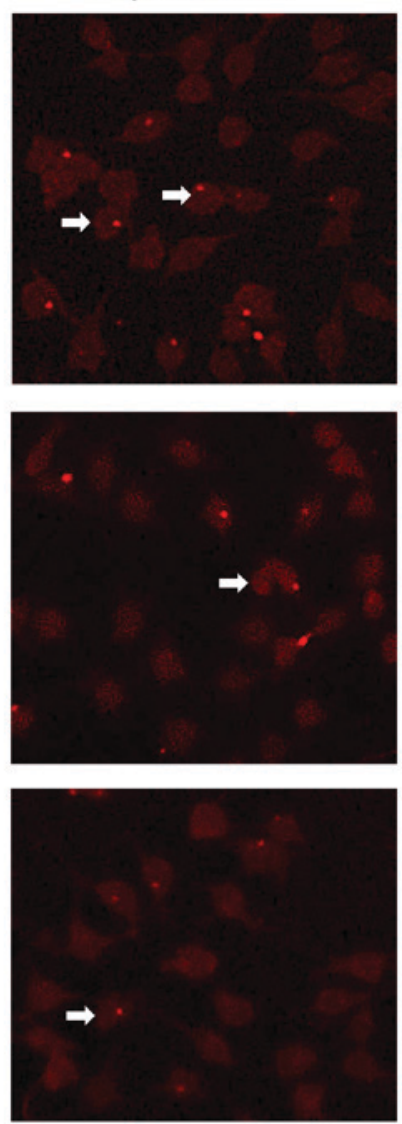

Acetylated- $\alpha$-tubulin
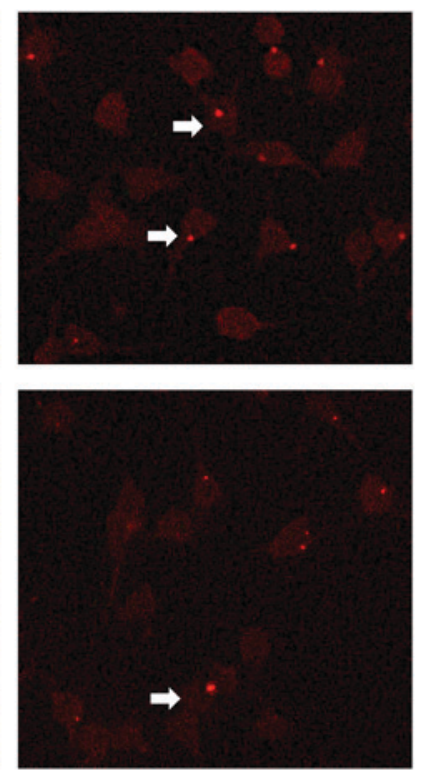
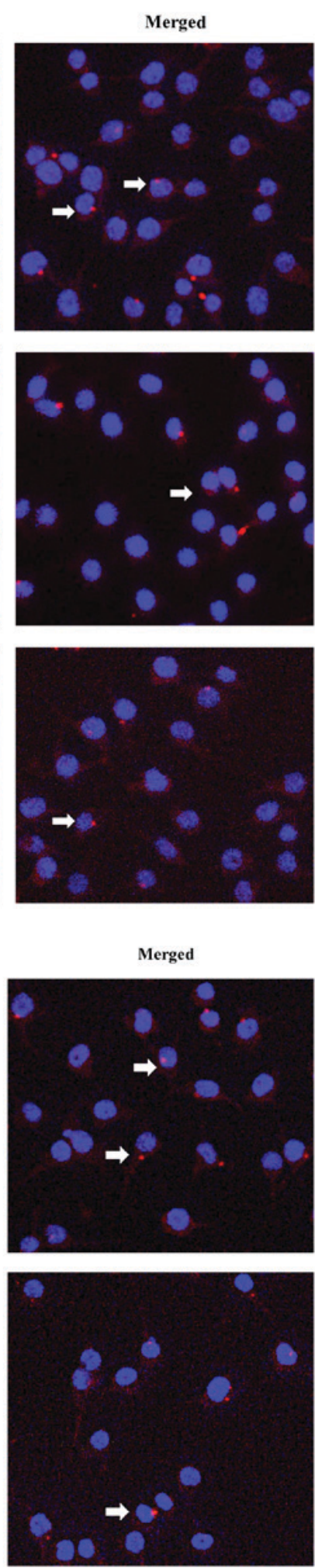

Figure 6. Treatment with the ERK inhibitor PD0325901 or the FGFR inhibitor BGJ398, and IFT88 knockdown suppressed the formation of primary cilia in murine ATDC5 chondrocytes. ATDC5 cells were cultured in the presence or absence of (A) PD0325901 or BGJ398 for 24 h, or (B) transfected with negative control or IFT88-targeting siRNA for $72 \mathrm{~h}$. The cells were then stained with an anti-acTub (red) antibody to detect primary cilia. DAPI was used to visualize the nuclei (blue). PD0325901 or BGJ398 treatment and IFT88 knockdown decreased the number of ciliated cells (white arrows) compared with the control group. Magnification, x200. ERK, extracellular signal-regulated protein kinase; FGF, fibroblast growth factor; FGFR, FGF receptor; IFT88, intraflagellar transport protein 88; si, small interfering; acTub, acetylated $\alpha$-tubulin.

present results demonstrated that bFGF treatment enhanced the phosphorylation of ERK, as indicated by western blot analysis of p-ERK expression (Fig. 2). Conversely, treatment with PD0325901 significantly downregulated the protein expression levels of p-ERK in ATDC5 cells, and suppressed IFT88 protein expression (Fig. 2B), however, no differences 

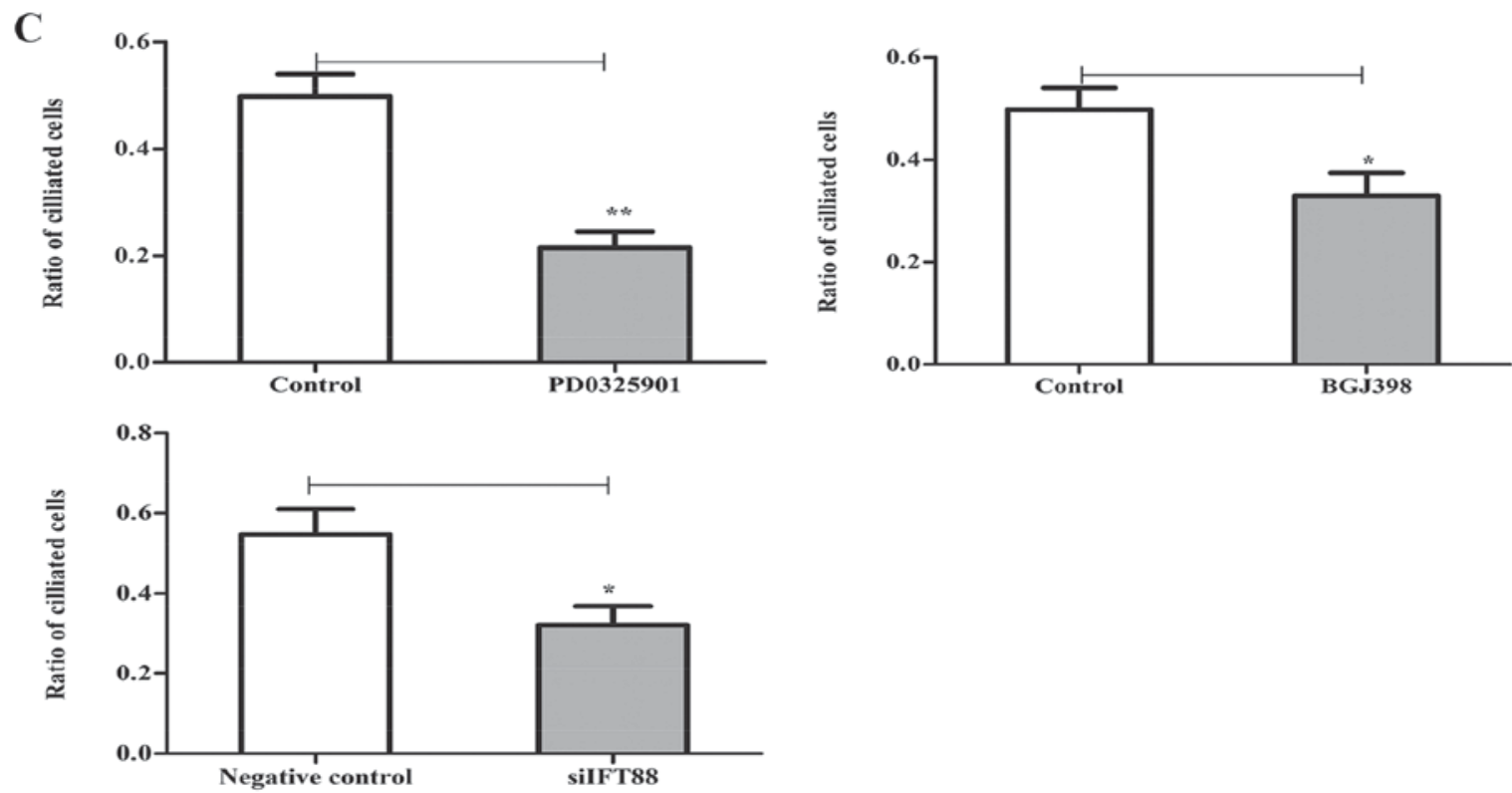

Figure 6. Continued. Treatment with the ERK inhibitor PD0325901 or the FGFR inhibitor BGJ398, and IFT88 knockdown suppressed the formation of primary cilia in murine ATDC5 chondrocytes. (C) The percentage of ciliated cells was calculated in 10 randomly selected fields of view and $>100$ cells were counted in each field. Data are expressed as the mean \pm standard deviation. ${ }^{*} \mathrm{P}<0.05,{ }^{* *} \mathrm{P}<0.01$, as indicated. ERK, extracellular signal-regulated protein kinase; FGF, fibroblast growth factor; FGFR, FGF receptor; IFT88, intraflagellar transport protein 88; si, small interfering; acTub, acetylated $\alpha$-tubulin.

were evident across total ERK expression levels. bFGF treatment in the presence of PD0325901 appeared to upregulate the expression of IFT88; however, the effect was significantly reduced when compared with bFGF treatment alone (Fig. 2B). A similar trend was revealed in $\mathrm{p}$-ERK protein expression levels, suggesting that PD0325901 may reduce the expression of IFT88 by inhibiting ERK phosphorylation. These findings suggested that the ERK signaling pathway may be involved in the regulation of IFT88 expression by bFGF in chondrocytes in vitro.

Inhibition of the FGF receptor (FGFR) suppresses IFT88 protein expression in ATDC5 cells. To investigate the effects of FGFR inhibition on IFT88 protein expression, ATDC5 cells were treated with the FGFR inhibitor BGJ398. BGJ398 primarily inhibits FGFR1, FGFR2 and FGFR3. The present findings revealed that BGJ398 downregulated the protein expression levels of IFT88 in ATDC5 cells, which was accompanied by a decrease in p-ERK expression levels (Fig. 3). These findings suggested that FGFR may participate in the molecular mechanisms underlying the regulation of IFT88 expression in chondrocytes.

Knockdown of IFT88 downregulates the protein expression of p-ERK in ATDC5 cells. To investigate the effects of IFT88 downregulation on the mitogen-activated protein kinase (MAPK)/ERK signaling pathway, RNA interference was used to silence IFT88 expression in ATDC5 cells. The efficacy of IFT88 siRNA transfection was confirmed (Fig. 4A), and the protein expression levels of IFT88, p-ERK and ERK were evaluated using western blot analysis. IFT88 knockdown was revealed to suppress the protein expression of IFT88 and p-ERK in ATDC5 cells, whereas it had no effect on ERK protein expression (Fig. 4B and C). These findings suggested that the MAPK/ERK signaling pathway may be involved in the regulation of IFT88 expression in ATDC5 cells.

bFGF exposure increases the number of ATDC5 cells exhibiting primary cilia. To investigate the effects of bFGF on the development of primary cilia, the presence of primary cilia in ATDC5 cells was detected using an acTub antibody. Following staining with the anti-acTub antibody, primary cilia were visualized as red acetylated $\alpha$-tubulin-positive structures in ATDC5 cells under a fluorescence microscope. bFGF exposure increased the percentage of ciliated cells compared with the control group (Fig. 5). bFGF treatment increased the number of ciliated cells by $\sim 80 \%$, suggesting that the bFGF-mediated regulation of IFT88 expression may be involved in the development of primary cilia in chondrocytes.

PD0325901 or BGJ398 treatment and IFT88 knockdown decreases the number of ciliated ATDC5 cells. To further investigate the effects of IFT88 downregulation on primary cilia development, primary cilia were visualized in ATDC5 cells using an anti-acTub antibody. Treatment with PD0325901 or BGJ398, and transfection with IFT88 siRNA, resulted in fewer ciliated cells compared with the control group (Fig. 6). These findings suggested that the regulation of IFT88 expression, and MAPK/ERK- and FGFR-mediated pathways may be involved in primary cilium maintenance.

bFGF treatment upregulates IFT88 expression and promotes primary cilium development in primary chondrocytes. To investigate whether primary chondrocytes may exhibit a similar response to bFGF to ATDC5 cells in vitro, the mRNA and protein expression of IFT8 8 was assessed in primary 

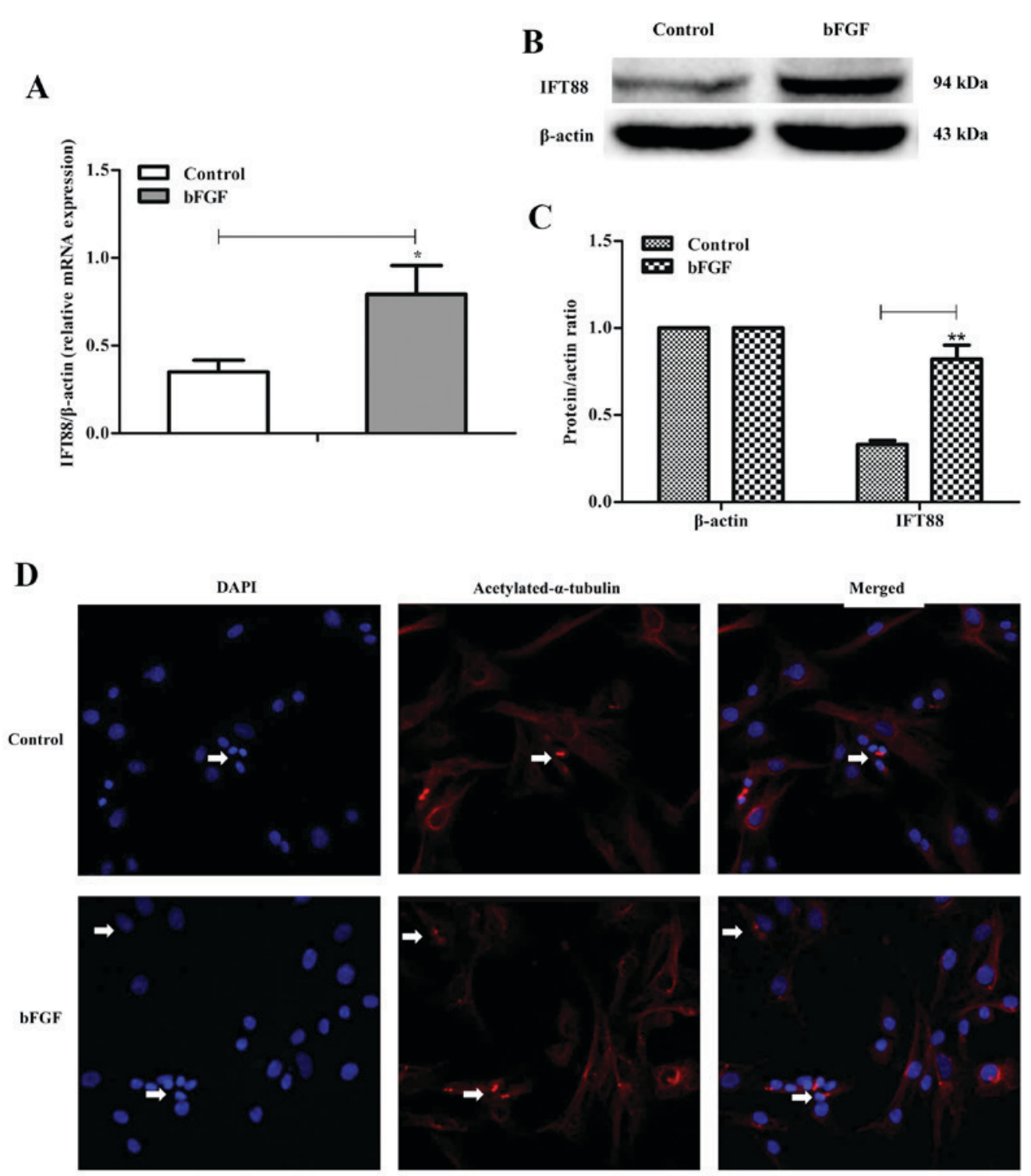

$\mathbf{E}$

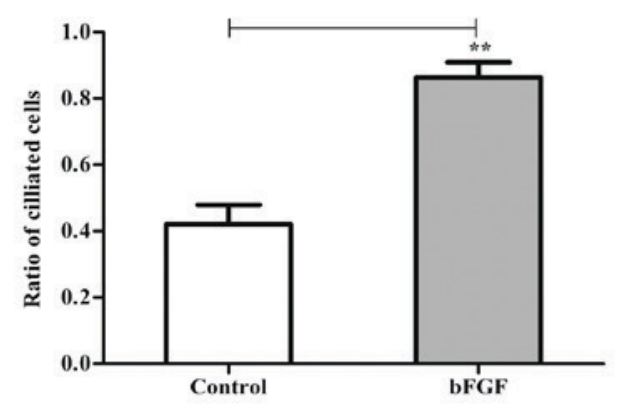

Figure 7. bFGF treatment upregulated the mRNA and protein expression of IFT88 and increased the number of ciliated cells in primary cultured chondrocytes Primary chondrocytes were cultured in the presence or absence of $5 \mathrm{ng} / \mathrm{ml} \mathrm{bFGF}$ for $24 \mathrm{~h}$. (A) mRNA expression levels of IFT88 were assessed using reverse transcription-quantitative polymerase chain reaction. (B and C) IFT88 protein expression levels were detected using western blot analysis. (D and E) The percentage of ciliated chondrocytes was determined in 10 randomly selected fields of view following staining with an anti-acTub antibody (>100 cells were counted/field; magnification, $\mathrm{x} 200$ ). Data are expressed as the mean \pm standard deviation of 3 independent experiments. ${ }^{*} \mathrm{P}<0.05,{ }^{* *} \mathrm{P}<0.01$, as indicated. bFGF, basic fibroblast growth factor; IFT88, intraflagellar transport protein 88; acTub, acetylated $\alpha$-tubulin.

chondrocytes. Primary chondrocytes were prepared from newborn mice and treated with bFGF at a concentration of $5 \mathrm{ng} / \mathrm{ml}$ for $24 \mathrm{~h}$. bFGF treatment upregulated the IFT88 mRNA and protein expression in primary chondrocytes (Fig. 7A-C). An immunofluorescence assay was used to evaluate the effects of exogenous bFGF on cilium development, and results 
revealed that bFGF increased the number of ciliated cells compared with the control group (Fig. 7D and E).

\section{Discussion}

The IFT protein complex is essential for the formation and maintenance of primary cilia in eukaryotic cells $(9,11,12)$; however, the molecular mechanisms involved in the effects of IFT complexes on cilia remain to be elucidated. In addition, little is known regarding the regulation of IFT proteins by cytokines in vivo or in vitro, and the effects of cytokines on cilium formation.

In the present study, exogenous bFGF was demonstrated to increase the mRNA and protein expression of IFT88 in ATDC5 chondrocytes in vitro. It is possible that the upregulated expression of IFT88 may potentiate the function of the IFT system in chondrocytes, through the amplification of mechanical stimulation and sensory perception of the extracellular microenvironment (6-8). These processes have been reported to contribute to the regulation of cartilage development $(19,20)$. A depletion of primary cilia has been demonstrated in the articular cartilage of Col2 $\alpha \mathrm{Cre}$; IFT88 ${ }^{\mathrm{A} / \mathrm{fl}}$ mice, which resulted in abnormal articular cartilage development (21). The cartilage of Col2 $\alpha$ Cre; IFT8 $8^{\mathrm{A} / \mathrm{f}}$ mice was thicker, had increased cell density and exhibited enhanced expression of osteoarthritic markers, including matrix metalloproteinase-13, disintegrin-like and metalloprotease with thrombospondin type 1 motif 5, collagen $\mathrm{X}$ and runt-related transcription factor 2 (21).

The findings of the present study suggested that bFGF may upregulate the protein expression of IFT88 through the MAPK/ERK signaling pathway in chondrocytes. Following treatment with the ERK inhibitor PD0325901, the FGFR inhibitor BGJ398, or IFT88-targeting siRNA, the protein expression levels of p-ERK appeared to be downregulated. These findings suggested that the IFT88 and MAPK/ERK pathways may be closely associated in chondrocytes; however, further studies are required to investigate the specific targets of the MAPK/ERK pathway and its downstream effects in primary cilia.

Murine chondrocytes express all FGFR subtypes (FGFR1-4); however, bFGF treatment has been reported to significantly induce the expression of FGFR3 $(22,23)$, which exerted anabolic effects in murine chondrocytes. In the present study, the FGFR inhibitor BGJ398 suppressed the expression of IFT88 in murine chondrocytes, thus suggesting that the IFT88 expression regulation process may involve FGFR regulation. It is of note that bFGF in human articular cartilage has been suggested to exert opposite roles compared with in murine cartilage, and FGF has been reported to activate catabolic processes primarily via FGFR1 signaling in human cartilage $(24,25)$. Considered together, these findings suggested that the effects of bFGF may be species-dependent.

Primary cilia have a microtubule-based infrastructure, which consists of 9 pairs of peripheral microtubules without a central microtubule, and can detect alterations in mechanical and biochemical stimulation from the extracellular milieu $(26,27)$. In addition, it has been suggested that cilia may be essential for the development and progression of tumors (28). In the present study, IFT88 expression was downregulated in ATDC5 chondrocytes in vitro, through the inhibition of ERK and FGFR signaling. Following siRNA-mediated knockdown, IFT88 downregulation resulted in the suppression of primary cilia development in chondrocytes. These findings suggested that IFT88 may be an essential factor for the formation and maintenance of primary cilia. To the best of our knowledge, the present study demonstrated for the first time that bFGF enhanced the mRNA and protein expression of IFT88, which in turn may promote primary cilia formation in chondrocytes. These findings propose a novel function for bFGF in chondrocytes.

\section{Acknowledgements}

The present study was supported by the National Natural Science Foundations of China (grant nos. 81371915 and 81572094).

\section{References}

1. Seeley ES and Nachury MV: The perennial organelle: Assembly and disassembly of the primary cilium. J Cell Sci 123: 511-518, 2010.

2. Kim S and Dynlacht BD: Assembling a primary cilium. Curr Opin Cell Biol 25: 506-511, 2013.

3. Satir P and Christensen ST: Overview of structure and function of mammalian cilia. Annu Rev Physiol 69: 377-400, 2007.

4. Enuka Y, Hanukoglu I, Edelheit O, Vaknine H and Hanukoglu A: Epithelial sodium channels $(\mathrm{ENaC})$ are uniformly distributed on motile cilia in the oviduct and the respiratory airways. Histochem Cell Biol 137: 339-353, 2012.

5. Tobin JL and Beales PL: The nonmotile ciliopathies. Genet Med 11: 386-402, 2009

6. Hoey DA, Tormey S, Ramcharan S, O'Brien FJ and Jacobs CR: Primary cilia-mediated mechanotransduction in human mesenchymal stem cells. Stem Cells 30: 2561-2570, 2012.

7. Ishikawa $\mathrm{H}$ and Marshall WF: Ciliogenesis: Building the cell's antenna. Nat Rev Mol Cell Biol 12: 222-234, 2011.

8. Muhammad H, Rais Y, Miosge N and Ornan EM: The primary cilium as a dual sensor of mechanochemical signals in chondrocytes. Cell Mol Life Sci 69: 2101-2107, 2012.

9. Rosenbaum JL and Witman GB: Intraflagellar transport. Nat Rev Mol Cell Biol 3: 813-825, 2002.

10. Mizuno N, Taschner M, Engel BD and Lorentzen E: Structural studies of ciliary components. J Mol Biol 422: 163-180, 2012.

11. Sung $\mathrm{CH}$ and Leroux MR: The roles of evolutionarily conserved functional modules in cilia-related trafficking. Nat Cell Biol 15: 1389-1397, 2013.

12. Pazour GJ, Baker SA, Deane JA, Cole DG, Dickert BL, Rosenbaum JL, Witman GB and Besharse JC: The intraflagellar transport protein, IFT88, is essential for vertebrate photoreceptor assembly and maintenance. J Cell Biol 157: 103-113, 2002.

13. Pazour GJ, Dickert BL, Vucica Y, Seeley ES, Rosenbaum JL, Witman GB and Cole DG: Chlamydomonas IFT88 and its mouse homologue, polycystic kidney disease gene tg737, are required for assembly of cilia and flagella. J Cell Biol 151: 709-718, 2000.

14. Friedl A, Chang Z, Tierney A and Rapraeger AC: Differential binding of fibroblast growth factor-2 and -7 to basement membrane heparin sulfate: Comparison of normal and abnormal human tissues. Am J Pathol 150: 1443-1455, 1997.

15. Okada-Ban M, Thiery JP and Jouanneau J: Fibroblast growth factor-2. Int J Biochem Cell Biol 32: 263-267, 2000.

16. Song H, Kwon K, Lim S, Kang SM, Ko YG, Xu Z, Chung JH, Kim BS, Lee H, Joung B, et al: Transfection of mesenchymal stem cells with the FGF-2 gene improves their survival under hypoxic conditions. Mol Cells 19: 402-407, 2005.

17. Ng EW and Adamis AP: Targeting angiogenesis, the underlying disorder in neovascular age-related macular degeneration. Can J Ophthalmol 40: 352-368, 2005.

18. Livak KJ and Schmittgen TD: Analysis of relative gene expression data using real-time quantitative PCR and the 2(-Delta Delta C(T)) method. Methods 25: 402-408, 2001. 
19. McGlashan SR, Cluett EC, Jensen CG and Poole CA: Primary cilia in osteoarthritic chondrocytes: From chondrons to clusters. Dev Dyn 237: 2013-2020, 2008.

20. Wann AK, Zuo N, Haycraft CJ, Jensen CG, Poole CA, McGlashan SR and Knight MM: Primary cilia mediate mechanotransduction through control of ATP-induced $\mathrm{Ca} 2^{+}$signaling in compressed chondrocytes. FASEB J 26: 1663-1671, 2012.

21. Chang CF, Ramaswamy G and Serra R: Depletion of primary cilia in articular chondrocytes result in reduced Gli3 repressor to activator ratio, increased Hedgehog signaling, and symptoms of early osteoarthritis. Osteoarthritis Cartilage 20: 152-161, 2012.

22. Li X, Ellman MB, Kroin JS, Chen D, Yan D, Mikecz K, Ranjan KC, Xiao G, Stein GS, Kim SG, et al: Species-specific biological effects of FGF-2 in articular cartilage: Implication for distinct roles within the FGF receptor family. J Cell Biochem 113: 2532-2542, 2012.

23. Chia SL, Sawaji Y, Burleigh A, McLean C, Inglis J, Saklatvala J and Vincent T: Fibroblast growth factor 2 is an intrinsic chondroprotective agent that suppresses ADAMTS-5 and delays cartilage degradation in murine osteoarthritis. Arthritis Rheum 60: 2019-2027, 2009.
24. Yan D, Chen D, Cool SM, Van Wijnen AJ, Mikecz K, Murphy G and Im HJ: Fibroblast growth factor receptor 1 is principally responsible for fibroblast growth factor 2-induced catabolic activities in human articular chondrocytes. Arthritis Res Ther 13: R130, 2011.

25. Yan D, Chen D and Im HJ: Fibroblast growth factor-2 promotes catabolism via FGFR1-Ras-Raf-MEK1/2-ERK1/2 axis that coordinates with the PKC $\delta$ pathway in human articular chondrocytes. J Cell Biochem 113: 2856-2865, 2012.

26. Pazour GJ and Witman GB: The vertebrate primary cilium is a sensory organelle. Curr Opin Cell Biol 15: 105-110, 2003.

27. Bloodgood RA: Sensory reception is an attribute of both primary cilia and motile cilia. J Cell Sci 123: 505-509, 2010.

28. Xiang W, Jiang T, Guo F, Gong C, Yang K, Wu Y, Huang X, Cheng W and Xu K: Hedgehog pathway inhibitor-4 suppresses malignant properties of chondrosarcoma cells by disturbing tumor ciliogenesis. Oncol Rep 32: 1622-1630, 2014. 